\title{
Associations in tumor infiltrating lymphocytes between clinicopathological factors and clinical outcomes in estrogen receptor-positive/human epidermal growth factor receptor type 2 negative breast cancer
}

YUICHIRO MIYOSHI ${ }^{1}$, TADAHIKO SHIEN ${ }^{1}$, AKIKO OGIYA $^{2}$, NAOKO ISHIDA $^{3}$, KIEKO YAMAZAKI ${ }^{2}$, RIE HORII $^{4}$, YOSHIYA HORIMOTO ${ }^{5}$, NORIKAZU MASUDA ${ }^{6}$, HIROYUKI YASOJIMA ${ }^{6}$, TOUKO INAO ${ }^{7}$, TOMOFUMI OSAKO $^{8,11}$, MASATO TAKAHASHI ${ }^{9}$, NOBUMOTO TOMIOKA ${ }^{9}$, YUMI WANIFUCHI-ENDO ${ }^{10}$, MITSUCHIKA HOSODA $^{3}$, HIROYOSHI DOIHARA ${ }^{1}$ and HIROKO YAMASHITA ${ }^{3}$

${ }^{1}$ Department of Breast and Endocrine Surgery, Okayama University Hospital, Okayama 700-8558;

${ }^{2}$ Department of Breast Surgical Oncology, Cancer Institute Hospital, Japanese Foundation for Cancer Research, Tokyo 135-8550; ${ }^{3}$ Department of Breast Surgery, Hokkaido University Hospital, Hokkaido 060-8648; ${ }^{4}$ Division of Pathology,

Cancer Institute Hospital, Japanese Foundation for Cancer Research, Tokyo 135-8550; ${ }^{5}$ Department of Breast Oncology, Juntendo University School of Medicine, Tokyo 113-8431; ${ }^{6}$ Department of Surgery, Breast Oncology, NHO Osaka National Hospital, Osaka 540-0006; ${ }^{7}$ Department of Breast and Endocrine Surgery, Graduate School of Medical Science Kumamoto University, Kumamoto $860-8556 ;{ }^{8}$ Department of Breast and Endocrine Surgery, Kumamoto City Hospital, Kumamoto 862-8505; ${ }^{9}$ Department of Breast Surgery, NHO Hokkaido Cancer Center, Hokkaido 003-0804; ${ }^{10}$ Department of Breast Surgery, Nagoya City University Graduate School of Medical Sciences, Nagoya 467-8601, Japan

Received June 17, 2018; Accepted November 29, 2018

DOI: $10.3892 / 01.2018 .9853$

\begin{abstract}
The value of assessing tumor infiltrating lymphocytes (TILs) in estrogen receptor (ER) positive/human epidermal growth factor receptor type 2 (HER2) negative
\end{abstract}

Correspondence to: Dr Tadahiko Shien, Department of Breast and Endocrinological Surgery, Okayama University Hospital, 2-5-1 Shikata-cho, Okayama 700-8558, Japan

E-mail: tshien@md.okayama-u.ac.jp

${ }^{11}$ Present address: Department of Breast Surgery, Kumamoto Shinto General Hospital, Kumamoto 862-8655, Japan

Abbreviations: TILs, tumor infiltrating lymphocytes; ER, estrogen receptor; PgR, progesterone receptor; HER2, human epidermal growth factor receptor 2; Ki67 LI, Ki67 labeling index; IRB, institutional review board; HE, hematoxylin-eosin; OR, odds ratio; CI, confidence interval; IDC-NST, invasive ductal carcinoma of no special type; $\mathrm{A}+\mathrm{T}$, anthracycline and taxane; A, anthracycline; $\mathrm{T}$, taxane; CMF, cyclophosphamide, methotrexate and fluorouracil; TAM, tamoxifen; TAM+LHRH, tamoxifen and luteinizing hormone-releasing hormone; $\mathrm{TAM} \rightarrow \mathrm{AI}$, tamoxifen followed by aromatase inhibitor; TAM+LHRH $\rightarrow \mathrm{AI}$, tamoxifen and luteinizing hormone-releasing hormone followed by aromatase inhibitor; AI, aromatase inhibitor; LHRH, luteinizing hormone-releasing hormone; EarR, early recurrence; LateR, late recurrence; NoR, no recurrence; LPBC, lymphocyte-predominant breast cancer; CT, chemotherapy; ET, endocrine therapy; CT+ET, chemotherapy + endocrine therapy breast cancer has yet to be determined. In the present study, a total of 184 cases with early distant recurrence detected within 5 years following the primary operation, 134 with late distant recurrence diagnosed following 5 years or longer and 321 controls without recurrence for $>10$ years following starting the initial treatment for ER-positive/HER2 negative breast cancer, registered in 9 institutions, were analyzed. The distributions of TILs and their clinical relevance were investigated. TIL distributions did not differ significantly among the early, late and no recurrence groups, employing a $30 \%$ cut-off point as a dichotomous variable. In those who had received adjuvant chemotherapy as well as endocrine therapy, a trend toward higher TIL proportions was detected when the early recurrence group was compared with the no recurrence group employing the $30 \%$ cut-off point $(\mathrm{P}=0.064)$. The TIL distributions were significantly associated with nodal metastasis $(\mathrm{P}=0.004)$, ER status $(\mathrm{P}=0.045)$, progesterone receptor $(\mathrm{PgR})$ status $(\mathrm{P}=0.002)$, tumor grade $(\mathrm{P}=0.021)$, and the Ki67 labeling index $(\mathrm{LI})(\mathrm{P}=0.002)$ in the no recurrence group and with the Ki67 LI in the recurrence groups ( $\mathrm{P}=0.002$ in early recurrence group, $\mathrm{P}=0.023$ in late recurrence group). High TIL distributions also predicted shorter survival time following the

Key words: breast cancer, estrogen receptor positive, human epidermal growth factor receptor type 2 negative, tumor infiltrating lymphocytes, prognosis 
detection of recurrence $(\mathrm{P}=0.026)$. However, these prognostic interactions were not significant in multivariate analysis $(\mathrm{P}=0.200)$. The present retrospective study demonstrated no significant interaction between TIL proportions and the timing of recurrence. However, higher TIL proportions were observed in breast cancer patients with aggressive biological phenotypes, which tended to be more responsive to chemotherapy. The clinical relevance of stromal TILs for identifying patients who would likely benefit from additional therapies merits further investigation in a larger patient population.

\section{Introduction}

Breast cancer is a heterogeneous disease, which is categorized into subtypes according to gene expressions and clinicopathological features $(1,2)$. Luminal subtype, which is one of the recognized subtypes, is characteristically estrogen receptor (ER) positive and human epidermal growth factor receptor type 2 (HER2) negative. Endocrine therapy is considered first, and then chemotherapy in high-risk groups, based on the pathological diagnosis which includes histological classification, tumor grade, the Ki67 labeling index (LI) and lymph node metastasis, as adjuvant systemic therapies $(3,4)$. However, some luminal tumors recur regardless of adjuvant therapy, which is a critical problem that must be overcome to improve patient survival (5).

The importance of tumor infiltrating lymphocytes (TILs) has increasingly been recognized in recent years (6-9). The host immune system appears to influence the development of breast carcinoma (10). In addition to these observations, chemotherapy might trigger recruitment of lymphocytes to tumor nests $(11,12)$. We also need to understand whether TILs have an important role in patients receiving endocrine therapy $(13,14)$. Therefore, we need to understand the biological features and functions of TILs in the breast cancer microenvironment. Many studies have compared TILs among breast cancers to determine their prognostic value. Abundant TILs in highly proliferative tumors such as triple negative breast cancer and HER2 positive breast cancer have been demonstrated $(15,16)$. In a recent study, marked TIL infiltration was found to be associated with better outcomes for patients with these subtypes (17-23). On the other hand, recruitment of TILs was also reported to have variable impacts on the outcomes of ER positive/HER2 negative breast cancers $(13,15,24-28)$. However, details of TIL distributions are lacking as there have been few studies focusing on the timing of recurrence. In addition, the relationships between TIL distributions and the efficacies of systemic therapies such as chemotherapy and endocrine therapy remain poorly understood.

We retrospectively collected data from ER positive/HER2 negative breast cancer cases with early and late distant recurrence and from patients who remained recurrence free for more than ten years, and then identified clinicopathological factors predicting early and late recurrence in ER-positive/HER2 negative breast cancer cases (29-31). We next investigated the biological and prognostic significance of TILs, by comparing these three groups. We compared proportions of TILs among these groups and investigated associations between TIL distributions and clinicopathological factors in each group.

\section{Materials and methods}

Cases and clinical samples. This retrospective multi-institution study was conducted as Scientific Research of the Japanese Breast Cancer Society $(29,30)$. We registered 223 consecutive patients with early distant recurrence and 149 consecutive patients with late distant recurrence of ER-positive/HER2 negative breast cancer, who had undergone breast surgery and/or neoadjuvant chemotherapy between January 2000 and December 2004, from nine institutions. These institutes were Okayama University, the Cancer Institute Hospital, the Japanese Foundation for Cancer Research, Hokkaido University, Juntendo University, National Health Organization (NHO) Osaka National Hospital, Kumamoto University, Kumamoto City Hospital, the NHO Hokkaido Cancer Center, and Nagoya City University. Early recurrence was diagnosed based on distant metastasis within 5 years, late recurrence as distant metastasis more than 5 years after initial treatment. For each late recurrence patient, in general, two age-matched patients free of recurrence for more than ten years were randomly selected using RAND in combination with Excel software at each institution. In total, 321 patients who had been recurrence free for more than ten years served as study controls. The study protocol was approved by the institutional review board (IRB) of each participating institution and conformed to the guidelines of the 1996 Declaration of Helsinki. Opting out and a waiver of informed consent were options, as anonymized archival specimens were used in this retrospective study.

Expressions of ER, PgR, HER2, and Ki67 LI were centrally assessed employing immunohistochemistry. HER2-positive tumors were excluded from this study. The details were documented in our previous report (30).

Hematoxylin and eosin (H\&E) stained sections were available from 639 of the registered patients (early recurrence: $n=184$, late recurrence: $n=134$, no recurrence: $n=321$ ). In these cases with available sections, TIL proportions were compared among the three groups. We also assessed the relationships between TIL proportions and other clinicopathological features. Moreover, the relationship between TIL proportions and survival time after recurrence was evaluated for both groups with recurrent disease, i.e., both late and early recurrence.

Evaluation of TILs. H\&E-stained sections were utilized for evaluation of TILs. The percentages of stromal lymphocytes, serving as a predefined criterion in Denkert's et al and Loi's et al reports $(8,25,32)$, were evaluated by two observers. Stromal TILs were measured as the percentage of immune cells in stromal tissue within the tumor that showed a mononuclear immunological infiltrate (Fig. 1). Heterogeneous distributions were documented in almost all of the sections examined. Therefore, hot spots, cold spots, and Tertiary Lymphoid Structure were not taken into consideration in any of the measurements conducted; instead, one representative area was selected and evaluated. The findings were categorized according to three possible cut-off points for TIL proportions $(10,30$, and $50 \%)$.

Statistical analysis. Differences in clinicopathological data were compared between cases with and without recurrence 
employing the Chi-square test. The Chi square test was utilized when investigating associations between the TIL distribution and clinicopathological features in each group. The Kaplan-Meier method was used to estimate survival duration from the time-point of recurrence detection. Differences between overall survival curves were determined with the log-rank test. For both univariate and multivariate analyses, Cox regression was used to evaluate the influences of the variables on survival time. All of the data were analyzed employing JMP 11.0.0 (SAS Institute Inc., Cary, NC, USA) statistical software. A value of $\mathrm{P}<0.05$ was considered to indicate a statistically significant difference.

\section{Results}

Patient characteristics. Patient characteristics are presented in Table I. The median follow-up durations were 72 (range, 14-179), 133 (range, 67-177) and 128 (range, 57-179) months in the early, late and no recurrence groups, respectively. During follow-up of these 639 patients, $69.5 \%$ (128/184) of those with early recurrence and $31.3 \%(42 / 134)$ of those with late recurrence died of breast cancer. The histology was invasive ductal carcinoma in $94.0 \%$ $(173 / 184), 93.2 \%(125 / 134)$ and $93.1 \%$ (299/321) of the early, late, and no recurrence groups, respectively. The recurrences were local in $20.1 \%$ (37/184) of the early recurrence group and in $28.3 \%$ (38/134) of the late recurrence group. Adjuvant endocrine therapy alone had been administered to $28.8 \%$ (53/184) of the early recurrence cases, $41.0 \%(55 / 134)$ of the late recurrence cases, and $56.0 \%(180 / 321)$ of the controls, while $53.8 \%(99 / 184)$, $51.4 \%$ (69/134) and 32.3\% (104/321), respectively, received both adjuvant chemotherapy and endocrine therapy. The adjuvant chemotherapy consisted mainly of anthracyclines and/or taxanes.

Distributions of TILs. The TIL distributions are shown in Table II. Percentages of TILs did not differ significantly among the three groups $(\mathrm{P}=0.556)$. In previous reports, various cut-off points were utilized $(10,35,50$ and $60 \%)(14,25,27,28,33)$. However, there are as yet no standardized cut-off points. We selected a $30 \%$ cut-off point from among the potential values because there were few cases with TIL proportions lower than $10 \%$ or more than $50 \%$ in our study. We thus conducted the following analyses employing $30 \%$ as the cut-off point.

In each case, various adjuvant therapies had been administered. Therefore, we investigated the TIL distributions according to the presence of adjuvant chemotherapy and endocrine therapy. The odds ratios for recurrence with high TILs are presented in Table III. There were no significant interactions between TIL proportions and the time of recurrence in any of the subgroups. In those who had received adjuvant chemotherapy as well as endocrine therapy, a trend toward higher TIL proportions was detected when the early recurrence group was compared with the no recurrence group employing the $30 \%$ cut-off point $(\mathrm{P}=0.064)$.

Associations of TIL proportions with clinicopathological factors. We assessed whether the proportion of TILs was associated with clinicopathological factors (Table IV). TILs in breast cancer specimens correlated significantly with the Ki67 LI $(P=0.002)$ in the early recurrence group. Nodal metastasis $(\mathrm{P}=0.008)$, tumor grade $(\mathrm{P}=0.008)$, and Ki67 LI $(\mathrm{P}=0.023)$
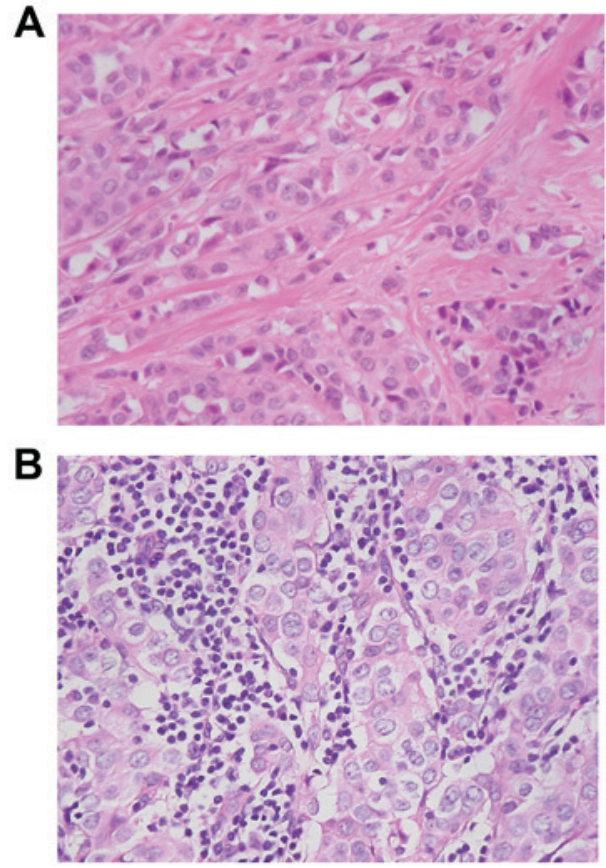

Figure 1. Hematoxylin and eosin staining of TILs in breast tumor cells. Among stromal areas within tumor nests, a small number of TILs ranging from 10 to $29 \%$ were identified in (A), but in (B) TIL proportions exceeded $50 \%$ (magnification, $\mathrm{x} 400$ ). TILs, tumor infiltrating lymphocytes.

showed significant associations with the proportion of TILs in the late recurrence group. Nodal metastasis $(\mathrm{P}=0.004), \mathrm{ER}$ status $(\mathrm{P}=0.045), \mathrm{PgR}$ status $(\mathrm{P}=0.002)$, tumor grade $(\mathrm{P}=0.021)$, and Ki67 LI $(\mathrm{P}=0.002)$ showed significant associations with the proportion of TILs in the no recurrence group.

Survival time after distant recurrence. We analyzed 318 cases (early recurrence: 184 (58\%), late recurrence: $134(42 \%)$ ) to compare survival duration from the time of recurrence detection between the cases with high and low TIL proportions (Fig. 2). The median follow-up duration from the detection of recurrence until death due to breast cancer was 39 (0-141) months in the early recurrence and $34(0-89)$ months in the late recurrence group. The Kaplan-Meier method revealed a significant difference between these two groups in TIL distributions ( $\mathrm{P}=0.026)$ (Fig. 2). Moreover, the Kaplan-Meier method revealed a trend for higher TIL proportions in the early recurrence group $(\mathrm{P}=0.080)$, while there was no difference in the late recurrence group $(\mathrm{P}=0.187)$. Univariate analysis of all cases with recurrence revealed TILs, nodal metastasis, and tumor grade to be significant prognostic factors. We selected significant parameters $(\mathrm{P}<0.20)$ from among various conventional confounding factors, and performed a multivariate analysis in which nodal metastasis, PgR, tumor grade, and Ki67 LI served as categorical variables. In this multivariate analysis, lymph node metastasis $(\mathrm{P}=0.027)$ was found to be an independent prognostic factor, while the proportion of TILs was not $(\mathrm{P}=0.200)$ (Table $\mathrm{V})$.

\section{Discussion}

In this retrospective study, the TIL proportions did not vary among recurrence patterns. However, among those 
Table I. Clinicopathological factors according to the time of recurrence.

\begin{tabular}{|c|c|c|c|c|c|c|}
\hline \multirow[b]{2}{*}{ Variable } & \multicolumn{3}{|c|}{ Number $(\%)$} & \multicolumn{3}{|c|}{ P-value } \\
\hline & $\operatorname{EarR}(\mathrm{n}=184)$ & LateR $(\mathrm{n}=134)$ & $\operatorname{NoR}(n=321)$ & EarR vs. NoR & LateR vs. NoR & EarR vs. LateR \\
\hline \multicolumn{7}{|l|}{ Age (years) } \\
\hline$\leqq 50$ & $82(44.5)$ & $49(36.5)$ & $120(37.3)$ & 0.113 & 0.869 & 0.151 \\
\hline$>50$ & $102(55.4)$ & $85(63.4)$ & $201(62.6)$ & & & \\
\hline \multicolumn{7}{|l|}{ Menopausal status } \\
\hline Post- & $90(48.9)$ & $81(60.4)$ & $171(53.2)$ & 0.345 & 0.159 & $0.041^{\mathrm{a}}$ \\
\hline Pre- & $94(51.0)$ & $53(39.5)$ & $150(46.7)$ & & & \\
\hline \multicolumn{7}{|l|}{ Bilateral breast cancer } \\
\hline Absent & $169(91.8)$ & $121(90.3)$ & $319(99.3)$ & $<0.001^{\mathrm{c}}$ & $<0.001^{\mathrm{c}}$ & 0.631 \\
\hline Present & $15(8.1)$ & $13(9.7)$ & $2(0.6)$ & & & \\
\hline \multicolumn{7}{|l|}{$\mathrm{cT}(\mathrm{mm})$} \\
\hline$\leqq 20$ & 49 (26.6) & $44(32.8)$ & $187(58.2)$ & $<0.001^{\mathrm{c}}$ & $<0.001^{\mathrm{c}}$ & 0.23 \\
\hline$>20$ & $135(73.3)$ & $90(67.1)$ & $134(41.7)$ & & & \\
\hline \multicolumn{7}{|l|}{$\mathrm{cN}$} \\
\hline Negative & $93(50.5)$ & 79 (58.9) & $267(83.1)$ & $<0.001^{\mathrm{c}}$ & $<0.001^{\mathrm{c}}$ & 0.136 \\
\hline Positive & $91(49.4)$ & $55(41.0)$ & $54(16.8)$ & & & \\
\hline \multicolumn{7}{|l|}{ Histological type } \\
\hline IDC-NST & $171(92.9)$ & $124(92.5)$ & $297(92.5)$ & 0.864 & 0.995 & 0.892 \\
\hline Others & $13(7.0)$ & $10(7.4)$ & $24(7.4)$ & & & \\
\hline \multicolumn{7}{|l|}{ Estrogen receptor $(\%)$} \\
\hline$<10$ & $16(8.7)$ & $10(7.4)$ & $27(8.4)$ & 0.396 & 0.367 & 0.915 \\
\hline $10-50$ & $57(30.9)$ & $43(32.0)$ & $82(25.5)$ & & & \\
\hline$\geqq 50$ & $111(60.3)$ & $81(60.4)$ & $212(66.0)$ & & & \\
\hline \multicolumn{7}{|l|}{ Progesterone receptor $(\%)$} \\
\hline$\leqq 20 \%$ & $85(46.2)$ & $59(44.0)$ & $133(41.4)$ & 0.298 & 0.609 & 0.701 \\
\hline$>20 \%$ & $99(53.8)$ & $75(55.9)$ & $188(58.5)$ & & & \\
\hline \multicolumn{7}{|l|}{ Tumor grade } \\
\hline 1 or 2 & $131(71.2)$ & $106(79.1)$ & $278(86.6)$ & $<0.001^{\mathrm{c}}$ & $<0.001^{\mathrm{c}}$ & 0.107 \\
\hline 3 & $53(28.8)$ & $28(20.9)$ & 43 (13.4) & & & \\
\hline \multicolumn{7}{|l|}{ Ki $67(\%)$} \\
\hline$\leqq 20$ & $136(73.9)$ & $114(85.0)$ & $265(82.5)$ & $<0.001^{\mathrm{c}}$ & 0.507 & $0.014^{\mathrm{a}}$ \\
\hline$>20$ & $48(26.0)$ & $20(14.9)$ & $56(17.4)$ & & & \\
\hline \multicolumn{7}{|l|}{ Local recurrence } \\
\hline Absent & $136(78.6)$ & $96(71.6)$ & 0 & & & \\
\hline Present & $37(21.3)$ & $38(28.3)$ & 0 & & & \\
\hline \multicolumn{7}{|l|}{ Surgical treatment } \\
\hline Total mastectomy & $117(63.5)$ & $78(58.2)$ & $106(33.0)$ & 0.076 & $<0.001^{\mathrm{c}}$ & 0.331 \\
\hline Partial mastectomy & $67(36.4)$ & $56(41.7)$ & $215(66.9)$ & & & \\
\hline \multicolumn{7}{|l|}{ Radiation therapy } \\
\hline Absent & $100(54.3)$ & $92(68.6)$ & $149(46.4)$ & $<0.001^{\mathrm{c}}$ & $<0.001^{\mathrm{c}}$ & $0.009^{b}$ \\
\hline Present & $84(45.6)$ & $42(31.3)$ & $172(53.5)$ & & & \\
\hline \multicolumn{7}{|l|}{ Adjuvant treatment } \\
\hline None & $13(7.0)$ & $4(2.9)$ & $30(9.3)$ & $<0.001^{\mathrm{c}}$ & $<0.001^{\mathrm{c}}$ & $0.021^{\mathrm{a}}$ \\
\hline Chemotherapy only & $19(10.3)$ & $6(4.4)$ & $7(2.1)$ & & & \\
\hline Endocrine therapy only & $53(28.8)$ & $55(41.0)$ & $180(56.0)$ & & & \\
\hline Combined therapy & $99(53.8)$ & $69(51.4)$ & $104(32.4)$ & & & \\
\hline \multicolumn{7}{|l|}{$\begin{array}{l}\text { Neoadjuvant } \\
\text { chemotherapy }\end{array}$} \\
\hline Absent & $168(91.3)$ & $131(97.7)$ & $305(95.3)$ & 0.076 & 0.198 & $0.011^{\mathrm{a}}$ \\
\hline Present & $16(8.7)$ & $3(2.2)$ & $15(4.6)$ & & & \\
\hline
\end{tabular}


Table I. Continued.

\begin{tabular}{|c|c|c|c|c|c|c|}
\hline \multirow[b]{2}{*}{ Variable } & \multicolumn{3}{|c|}{ Number (\%) } & \multicolumn{3}{|c|}{ P-value } \\
\hline & EarR (n=184) & LateR $(\mathrm{n}=134)$ & NoR $(n=321)$ & EarR vs. NoR & LateR vs. NoR & EarR vs. LateR \\
\hline \multicolumn{7}{|l|}{ Chemotherapy } \\
\hline $\mathrm{A}+\mathrm{T}$ & $54(29.3)$ & $22(16.4)$ & $42(13.0)$ & & & \\
\hline A & $39(21.2)$ & $31(23.1)$ & $40(12.4)$ & & & \\
\hline $\mathrm{T}$ & $6(3.2)$ & $7(5.2)$ & $13(4.0)$ & & & \\
\hline CMF & $17(9.2)$ & $12(8.9)$ & $13(4.0)$ & & & \\
\hline Others & $2(1.0)$ & $3(2.2)$ & $3(0.9)$ & & & \\
\hline None & $66(35.8)$ & $59(44.0)$ & $210(65.4)$ & & & \\
\hline \multicolumn{7}{|l|}{ Endocrine therapy } \\
\hline TAM & $61(33.1)$ & $34(25.3)$ & $68(21.1)$ & & & \\
\hline TAM+LHRH & $30(16.3)$ & $18(13.4)$ & 35 (10.9) & & & \\
\hline $\mathrm{TAM} \rightarrow \mathrm{AI}$ & $16(8.7)$ & $36(26.8)$ & $72(22.4)$ & & & \\
\hline $\mathrm{TAM}+\mathrm{LHRH} \rightarrow \mathrm{AI}$ & $1(0.5)$ & $3(2.2)$ & $15(4.6)$ & & & \\
\hline AI & $39(21.2)$ & $27(20.1)$ & $87(27.1)$ & & & \\
\hline LHRH & $5(2.7)$ & $6(4.4)$ & $7(2.1)$ & & & \\
\hline None & $32(17.3)$ & $10(7.4)$ & 37 (11.5) & & & \\
\hline
\end{tabular}

${ }^{\mathrm{a}} \mathrm{P}<0.05 ;{ }^{\mathrm{b}} \mathrm{P}<0.01 ;{ }^{\mathrm{c}} \mathrm{P}<0.001$. EarR, early recurrence; LateR, late recurrence; NoR, no recurrence; IDC-NST, invasive ductal carcinoma of no special type; A+T, anthracycline and taxane; A, anthracycline; T, taxane; CMF, cyclophosphamide, methotrexate, and fluorouracil; TAM, tamoxifen; TAM+LHRH, tamoxifen and luteinizing hormone-releasing hormone; TAM $\rightarrow$ AI, tamoxifen followed by aromatase inhibitor; $\mathrm{TAM}+\mathrm{LHRH} \rightarrow \mathrm{AI}$, tamoxifen and luteinizing hormone-releasing hormone followed by aromatase inhibitor; AI, aromatase inhibitor; LHRH, luteinizing hormone-releasing hormone; Ki67 Labeling index, Ki67 LI.

receiving chemotherapy and endocrine therapy, cases with higher TIL proportions tended to have fewer recurrences, though the difference did not reach statistical significance. In ER positive breast cancer, the significance of TILs for predicting recurrence appears to be minor, but those cases showing TIL recruitment might benefit from chemotherapy. Higher proportions of TILs were also observed in cancers showing markedly proliferative phenotypes. Further study is needed to identify associations among lymphocyte recruitment, aggressive features of the tumor and responsiveness to chemotherapy.

Many researchers have focused on the significance of differences in TIL proportions among breast cancer cases $(7,34)$. Methods for TIL evaluation varied among these studies. The methods used ranged from evaluation of H\&E sections for lymphocyte density and area, immune cell typing with immunohistochemistry and immune cell related transcriptome techniques (35-43). Many previous studies showed higher proportions of TILs to be observed in such de-differentiated tumors as triple negative breast cancer $(25,33,44)$. As to pathological examinations, tumor grade and Ki67 LI both correlated with higher TIL proportions (25). However, in those with triple negative breast cancer, higher TIL proportions also indicated a better prognosis according to several reports $(44,45)$, while one study found that in ER positive breast cancer accompanied by less recruitment of TILs than the triple negative subtype, the TIL proportions lacked prognostic significance (46). On the other hand, according to a few studies, TILs also correlated with the outcomes of patients with ER positive breast tumors $(14,33)$. The conflicting results obtained in these
Table II. Differences in the distributions of TIL proportions among recurrence pattern.

\begin{tabular}{lcccc}
\hline & \multicolumn{3}{c}{ Number (\%) } & \\
\cline { 2 - 4 } TIL positivity & EarR & LateR & NoR & P-value \\
\hline $0-10 \%$ & $11(5.9)$ & $4(2.9)$ & $17(5.3)$ & 0.556 \\
$10-30 \%$ & $151(82.0)$ & $113(84.3)$ & $261(81.3)$ & \\
$30-50 \%$ & $19(10.3)$ & $15(11.1)$ & $31(9.6)$ & \\
$50-100 \%$ & $3(1.6)$ & $2(1.4)$ & $12(3.7)$ & \\
\hline
\end{tabular}

EarR, early recurrence; LateR, late recurrence; NoR, no recurrence; TILs, tumor infiltrating lymphocytes.

studies may reflect different study populations and evaluation methods. We utilized H\&E-stained sections to evaluate representative densities of lymphocytes in the stromal area. The data obtained in this case control study of luminal breast tumors were analyzed by comparing TIL proportions among recurrence patterns. We also evaluated time of recurrence in a long-term follow-up study.

In our full study population, TILs showed no correlation with the timing of recurrence. However, among those receiving chemotherapy and endocrine therapy, cases with higher TIL proportions tended to experience fewer recurrences, though the difference did not reach statistical significance. We speculated that patients with breast tumors showing higher TIL 
Table III. Odds ratios between recurrence patterns.

\begin{tabular}{|c|c|c|c|c|c|c|}
\hline \multirow[b]{2}{*}{ Adjuvant therapy } & \multicolumn{3}{|c|}{$\begin{array}{l}\text { Number with high } \\
\text { TILs/total number }\end{array}$} & \multirow{2}{*}{$\begin{array}{c}\text { EarR vs. NoR } \\
\text { OR (CI) P-value }\end{array}$} & \multirow{2}{*}{$\frac{\text { LateR vs. NoR }}{\text { OR (CI) P-value }}$} & \multirow{2}{*}{$\frac{\text { EarR vs. LateR }}{\text { OR (CI) P-value }}$} \\
\hline & EarR & LateR & NoR & & & \\
\hline All & $22 / 184$ & $17 / 134$ & $43 / 321$ & $\begin{array}{c}0.877(0.499-1.505) \\
P=0.64\end{array}$ & $\begin{array}{c}0.939(0.502-1.686) \\
P=0.838\end{array}$ & $\begin{array}{c}0.934(0.476-1.859) \\
\mathrm{P}=0.844\end{array}$ \\
\hline $\mathrm{CT}+\mathrm{ET}$ & $13 / 99$ & $12 / 69$ & $24 / 104$ & $\begin{array}{c}0.503(0.234-1.041) \\
\mathrm{P}=0.064\end{array}$ & $\begin{array}{c}0.701(0.315-1.494) \\
P=0.363\end{array}$ & $\begin{array}{c}0.718(0.304-1.703) \\
P=0.447\end{array}$ \\
\hline ET & $5 / 53$ & $4 / 55$ & $15 / 180$ & $\begin{array}{c}1.145(0.358-3.129) \\
P=0.803\end{array}$ & $\begin{array}{c}0.862(0.237-2.502) \\
P=0.862\end{array}$ & $\begin{array}{c}1.328(0.332-5.641) \\
P=0.684\end{array}$ \\
\hline
\end{tabular}

EarR, early recurrence; LateR, late recurrence; NoR, no recurrence; OR, odds Ratio; CI, confidence interval; CT, chemotherapy; ET, endocrine therapy; CT+ET, chemotherapy + endocrine therapy; TILs, tumor infiltrating lymphocytes.

Table IV. Proportions of high and low TIL numbers in EarR, LateR, and NoR cases, in association with clinicopathological factors.

\begin{tabular}{|c|c|c|c|c|c|c|c|c|c|}
\hline \multirow[b]{3}{*}{ Variable } & \multicolumn{9}{|c|}{ Number of TILs (\%) } \\
\hline & \multicolumn{3}{|c|}{ EarR } & \multicolumn{3}{|c|}{ LateR } & \multicolumn{3}{|c|}{ NoR } \\
\hline & High & Low & P-value & High & Low & P-value & High & Low & P-value \\
\hline \multicolumn{10}{|l|}{ TILs } \\
\hline$\leqq 50$ & $12(54.5)$ & $70(43.2)$ & 0.317 & $3(17.6)$ & $46(39.3)$ & 0.068 & $16(37.2)$ & $104(37.4)$ & 0.979 \\
\hline$>50$ & $10(45.4)$ & $92(56.7)$ & & $14(82.3)$ & $71(60.6)$ & & $27(62.7)$ & $174(62.5)$ & \\
\hline \multicolumn{10}{|c|}{ Tumor size (mm) } \\
\hline$\leqq 20$ & $6(27.2)$ & $43(26.5)$ & 0.942 & $4(23.5)$ & $40(34.1)$ & 0.369 & $24(55.8)$ & $163(58.6)$ & 0.727 \\
\hline$>20$ & $16(72.7)$ & $119(73.4)$ & & $13(76.4)$ & $77(65.8)$ & & $19(44.1)$ & $115(41.3)$ & \\
\hline \multicolumn{10}{|c|}{ Lymph node metastases } \\
\hline Negative & $11(50.0)$ & $82(50.6)$ & 0.956 & $5(29.4)$ & $74(63.2)$ & $0.008^{\mathrm{b}}$ & $29(67.4)$ & $238(85.6)$ & 0.004 \\
\hline Positive & $11(50.0)$ & $80(49.3)$ & & $12(70.5)$ & $43(36.7)$ & & $14(32.5)$ & $40(14.3)$ & \\
\hline \multicolumn{10}{|c|}{ Estrogen receptor $(\%)$} \\
\hline$<10$ & $1(4.5)$ & $15(9.2)$ & 0.622 & $1(5.8)$ & $9(7.6)$ & 0.917 & $8(18.60)$ & $19(6.8)$ & $0.045^{\mathrm{a}}$ \\
\hline $10-50$ & $6(27.2)$ & $51(31.4)$ & & $5(29.4)$ & $38(32.4)$ & & $12(27.91)$ & $70(25.1)$ & \\
\hline$\geqq 50$ & $15(68.1)$ & $96(59.2)$ & & $11(64.7)$ & $70(59.8)$ & & $23(53.49)$ & $189(67.9)$ & \\
\hline \multicolumn{10}{|c|}{ Progesterone receptor $(\%)$} \\
\hline$\leqq 20 \%$ & $9(40.9)$ & $76(46.9)$ & 0.594 & $9(52.9)$ & $50(42.7)$ & 0.430 & $27(62.7)$ & $106(38.1)$ & $0.002^{\mathrm{b}}$ \\
\hline$>20 \%$ & $13(59.0)$ & $86(53.0)$ & & $8(47.0)$ & $67(57.2)$ & & $16(37.2)$ & $172(61.8)$ & \\
\hline \multicolumn{10}{|c|}{ Histological grade } \\
\hline 1 or 2 & $13(59.0)$ & $118(72.8)$ & 0.194 & $9(52.9)$ & $97(82.9)$ & $0.008^{\mathrm{b}}$ & $32(74.4)$ & $246(88.4)$ & $0.020^{\mathrm{a}}$ \\
\hline 3 & $9(40.9)$ & $44(27.1)$ & & $8(47.0)$ & $20(17.0)$ & & $11(25.5)$ & $32(11.5)$ & \\
\hline \multicolumn{10}{|l|}{ Ki $67(\%)$} \\
\hline$\leqq 20$ & $10(45.4)$ & $126(77.7)$ & $0.002^{\mathrm{b}}$ & $11(64.7)$ & $103(88.0)$ & $0.023^{\mathrm{a}}$ & $28(65.1)$ & $237(85.2)$ & $0.002^{\mathrm{b}}$ \\
\hline$>20$ & $12(54.5)$ & $36(22.2)$ & & $6(35.2)$ & $14(11.9)$ & & $15(34.8)$ & $41(14.7)$ & \\
\hline
\end{tabular}

${ }^{\mathrm{a}} \mathrm{P}<0.05$; ${ }^{\mathrm{b}} \mathrm{P}<0.01$. EarR, early recurrence; LateR, late recurrence; NoR, no recurrence; Ki67, Ki67 Labeling index; TILs, tumor infiltrating lymphocytes.

proportions might benefit more from chemotherapy. ER positive breast cancer patients received endocrine therapy, which was an important aspect of clinical management. Different adjuvant therapy modalities might make interpreting the 
Table V. Univariate and multivariate analyses for survival time from recurrence detection until mortality due to breast cancer.

\begin{tabular}{llll}
\hline & & \multicolumn{2}{c}{ Hazard ratio } \\
\cline { 2 - 4 } Variable & Univariate analysis & P-value & Multivariate analysis \\
\hline TILs $\leqq 30 />30$ & $1.598(1.028-2.386)$ & $0.037^{\mathrm{a}}$ & $1.348(0.847-2.072)$ \\
Age $($ years $) \leqq 50 />50$ & $1.160(0.856-1.581)$ & 0.338 & Not selected \\
Bilateral breast cancer: absent/present & $0.813(0.438-1.376)$ & 0.438 & Not selected \\
cT $\leqq 20 />20$ & $1.062(0.764-1.503)$ & 0.721 & Not selected \\
cN negative/positive & $1.500(1.109-2.032)$ & $0.008^{\mathrm{b}}$ & $1.412(1.039-1.921)$ \\
PgR $<20 / \geqq 20$ & $0.789(0.582-1.072)$ & 0.130 & $0.782(0.575-1.065)$ \\
Ki67 $<20 / \geqq 20$ & $1.270(0.888-1.781)$ & 0.184 & $1.176(0.806-1.681)$ \\
Tumor grade 1 or $2 / 3$ & $1.519(1.080-2.105)$ & $0.016^{\mathrm{a}}$ & $1.385(0.976-1.937)$ \\
\hline
\end{tabular}

${ }^{\mathrm{a}} \mathrm{P}<0.05 ;{ }^{\mathrm{b}} \mathrm{P}<0.01$. TILs, tumor infiltrating lymphocytes; PgR, progesterone receptor; Ki67, Ki67 Labeling index.

A

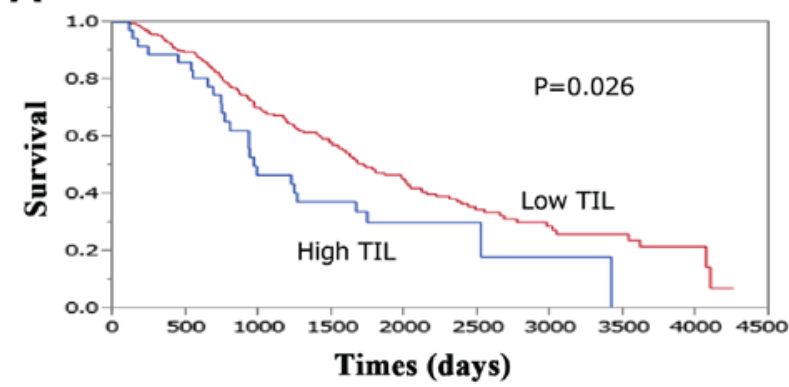

B

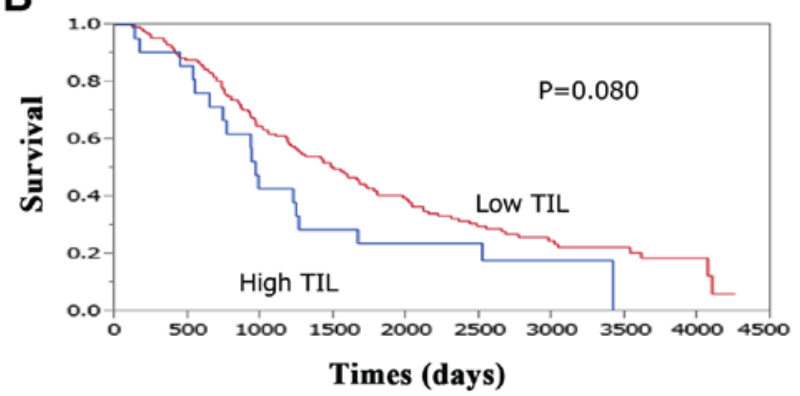

C

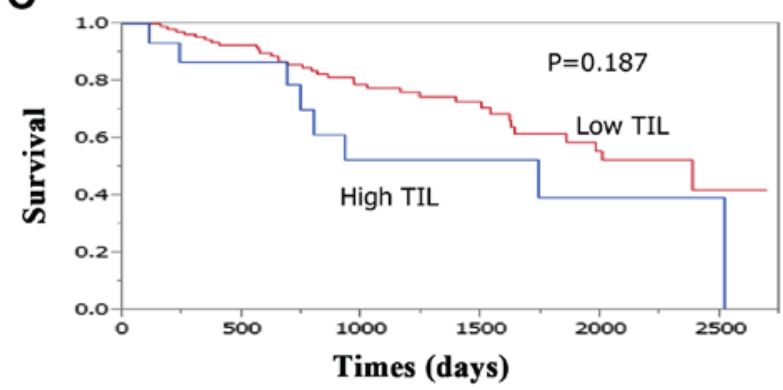

Figure 2. Survival time from recurrence detection until mortality due to breast cancer. There were significant differences in the (A) recurrence group as a whole. However, there was no significant difference in the (B) early recurrence group or in the (C) late recurrence group. TILs, tumor infiltrating lymphocytes.

roles of TILs difficult. Denkert et al (32) defined lymphocyte predominant breast cancer (LPBC) as showing evenly and widely distributed tumor lymphocytes in tumor nests (16).
LPBC was defined as a mean density of TILs of at least $50 \%$. However, most luminal breast cancers showed heterogeneous and low-density infiltration of lymphocytes into the stromal area. Therefore, identifying LPBC might not allow adequate evaluation of luminal breast tumors (16). Reliable methods for evaluating these heterogeneous distributions and the low density of TILs are as yet lacking. According to Vassiliki's report, investigation employing a $35 \%$ cut-off point as a binary parameter revealed an interaction between high TIL proportions and better outcomes, but this interaction was not statistically significant (33). According to Denkert's et al report, a high proportion of TILs is associated with a high pathological complete response rate (27). Jang and Kwon, employing a $10 \%$ cut-off value, reported high TIL proportions to be associated with better outcomes for luminal B (ER positive, HER2 negative, and higher Ki67) breast cancer patients who received adjuvant anthracycline (28). Higher TIL recruitment might make cancer cells more responsive to chemotherapy. The various cut-off points need to be investigated in terms of their significance when applied with various chemotherapy regimens. Studies utilizing ecological measurements and immune cell typing with immunohistochemistry have shown associations of lymphocytes, specifically their functions, with patient outcomes $(15,35,47-49)$. The value of H\&E-stained sections for determining lymphocyte density and area might be limited because the function of lymphocyte recruitment around tumor nests is not revealed by this method. Lymphocytes might promote or negatively regulate the growth of tumor nests. Flow cytometry, immunohistochemistry, and transcriptome analysis may be useful for determining the cell counts of specific lymphocyte populations. Further detailed studies, focusing on the function, extent and localization of tumor lymphocytes, are needed.

According to previous reports, high TIL proportions were found in aggressive breast cancer subtypes $(15,25)$. In our present study, in each group, TILs were identified in specimens from cases with a high Ki67 LI. Moreover, the TIL distribution correlated significantly with nodal metastasis, ER status, PgR status, tumor grade, and Ki67 LI in the no recurrence group. High TIL proportions correlated with rapid tumor growth, 
leading to death from breast cancer recurrence. Studies have demonstrated associations between high TIL proportions and poorer outcomes $(13,26,27)$. On the other hand, several reports have noted an association between TIL proportions and the effects of chemotherapy (15). We speculated that infiltration and accumulation of TILs might reflect both the aggressiveness and the fragility of cancer cells, suggesting that patients would benefit from cytotoxic agents. However, minor clusters of TILs in luminal breast tumors appear to have little, if any, role in recurrence and thus might not be important when considering various adjuvant settings. On the other hand, patients with breast tumors showing TIL recruitment might have a slightly poorer prognosis after recurrence due to the aggressive nature of these tumors. Luminal subtype tumors are characteristically ER positive and HER2 negative, reflecting heterogeneous breast cancer biology. Hormonal treatment is considered first, and then chemotherapy in high-risk groups, based on the pathological diagnosis which includes histological classification, tumor grade, Ki67 LI and lymph node metastasis. Therefore, we need to investigate the significance of TILs as a predictive factor for selecting therapies such as chemotherapy, endocrine therapy and molecular targeted therapy among prospective cohorts in a well-planned adjuvant setting. TILs may serve as a surrogate marker for systemic therapies. Additional translational research is also required to fully investigate the significance of potential TIL biomarkers.

Our study is limited by its retrospective, case control design. Therapy selections and intervals varied among physicians. Our results might thus have been affected by selection bias. Also, our sample was small. Therefore, our conclusions are inevitably somewhat controversial. Verification of our findings requires a prospective, well-planned study with a large cohort. In the present study, we utilized a predefined cut-off point to categorize the subgroups according to different TIL proportions. This cut-off point was selected from among potential cut-off value candidates based on our dataset. Therefore, this cut-off point might not be optimal. An optimal cut-off point needs to be established, in a future study, based on the details of TIL proportions as a continuous parameter.

In our present study, recruitment of TILs was more often observed in aggressive phenotypes, such as ER positive, HER2 negative breast cancer, but did not significantly predict recurrence. However, higher TIL proportions were observed in breast cancer patients with aggressive biological phenotypes which tended to be more responsive to chemotherapy. The significance of stromal TILs for identifying patients likely to benefit from additional therapies merits investigation in a large future study.

\section{Acknowledgements}

The authors would like to thank for Dr Bierta Barfod (Department of Neurosurgery, Katsuta Hospital, Ibaraki, Japan) for proofreading the manuscript.

\section{Funding}

The present study was supported by a Grant-in-Aid for Scientific Research from the Japanese Breast Cancer Society and the Health and Labour Sciences Research Expenses for
Commission, Applied Research for Innovative Treatment of Cancer, H26-applied-general-043 from the Ministry of Health, Labour and Welfare and the Practical Research for Innovative Cancer Control from the Japan Agency for Medical Research and Development, AMED (grant no. 17ck0106307h0001).

\section{Availability of data and materials}

The datasets used during the present study are available from the corresponding author upon reasonable request.

\section{Authors' contributions}

TS, AO, RH, YH, NM, TI, TO, MT, YWE and HYam designed the study. YM, TS, AO, NI, KY, RH, YH, NM, HYas, TI, TO, MT, NT, YWE, MH, HD, and HYam collected and assessed patient data. NI, MH, and HYam performed the immunohistochemical studies and evaluated the stained specimens. YM and TS evaluated TIL proportions in H\&E-stained sections. YM, HD and TS analyzed the patient data. YM, TS and HD drafted the manuscript. AO, NO, KY, RH, YH, NM, HYas, TI, TO, MT, NT, YWE, MH and HYam revised the manuscript. All authors read and approved the manuscript for submission.

\section{Ethics approval and consent to participate}

The study protocol was approved by the IRB of each participating institution. Patients were given the choice of opting out and an informed consent waiver was an option as anonymized archival specimens were used in this retrospective study.

\section{Patient consent for publication}

Not applicable.

\section{Competing interests}

The authors declare that they have no competing interests.

\section{References}

1. Mao XY, Lee MJ, Zhu J, Zhu C, Law SM and Snijders AM: Genome-wide screen identifies a novel prognostic signature for breast cancer survival. Oncotarget 8: 14003-14016, 2017.

2. Tsai M, Lo S, Audeh W, Qamar R, Budway R, Levine E, Whitworth P, Mavromatis B, Zon R, Oldham D, et al: Association of 70-gene signature assay findings with physicians' treatment guidance for patients with early breast cancer classified as intermediate risk by the 21-gene assay. JAMA Oncol 4: e173470, 2018.

3. Palmieri $\mathrm{C}$ and Jones A: The 2011 EBCTCG polychemotherapy overview. Lancet 379: 390-392, 2012.

4. Singh K, He X, Kalife ET, Ehdaivand S, Wang Y and Sung CJ: Relationship of histologic grade and histologic subtype with oncotype Dx recurrence score; retrospective review of 863 breast cancer oncotype Dx results. Breast Cancer Res Treat 168: 29-34, 2018.

5. Rouzier R, Perou CM, Symmans WF, Ibrahim N, Cristofanilli M, Anderson K, Hess KR, Stec J, Ayers M, Wagner P, et al: Breast cancer molecular subtypes respond differently to preoperative chemotherapy. Clin Cancer Res 11: 5678-5685, 2005.

6. Salgado R, Denkert C, Demaria S, Sirtaine N, Klauschen F, Pruneri G, Wienert S, Van den Eynden G, Baehner FL, Penault-Llorca F, et al: The evaluation of tumor-infiltrating lymphocytes (TILs) in breast cancer: Recommendations by an International TILs Working Group 2014. Ann Oncol 26: 259-271, 2015. 
7. Denkert C, von Minckwitz G, Brase JC, Sinn BV, Gade S, Kronenwett R, Pfitzner BM, Salat C, Loi S, Schmitt WD, et al: Tumor-infiltrating lymphocytes and response to neoadjuvant chemotherapy with or without carboplatin in human epidermal growth factor receptor 2-positive and triple-negative primary breast cancers. J Clin Oncol 33: 983-991, 2015.

8. Denkert C, Wienert S, Poterie A, Loibl S, Budczies J, Badve S Bago-Horvath Z, Bane A, Bedri S, Brock J, et al: Standardized evaluation of tumor-infiltrating lymphocytes in breast cancer: Results of the ring studies of the international immuno-oncology biomarker working group. Mod Pathol 29: 1155-1164, 2016.

9. Dieci MV, Radosevic-Robin N, Fineberg S, van den Eynden G, Ternes N, Penault-Llorca F, Pruneri G, D'Alfonso TM, Demaria S, Castaneda C, et al: Update on tumor-infiltrating lymphocytes (TILs) in breast cancer, including recommendations to assess TILs in residual disease after neoadjuvant therapy and in carcinoma in situ: A report of the international immuno-oncology biomarker working group on breast cancer. Semin Cancer Biol 52: 16-25, 2018.

10. Horimoto Y, Hayashi T and Arakawa A: Pathology of healing: What else might we look at? Cancer Med 5: 3586-3587, 2016.

11. Dieci MV, Arnedos M, Delaloge S and Andre F: Quantification of residual risk of relapse in breast cancer patients optimally treated. Breast 22 (Suppl 2): S92-S95, 2013

12. Demaria S, Volm MD, Shapiro RL, Yee HT, Oratz R, Formenti SC, Muggia F and Symmans WF: Development of tumor-infiltrating lymphocytes in breast cancer after neoadjuvant paclitaxel chemotherapy. Clin Cancer Res 7: 3025-3030, 2001

13. Blok EJ, Engels CC, Dekker-Ensink G, Meershoek-Klein Kranenbarg E, Putter H, Smit VTHBM, Liefers GJ, Morden JP, Bliss JM, Coombes RC, et al: Exploration of tumour-infiltrating lymphocytes as a predictive biomarker for adjuvant endocrine therapy in early breast cancer. Breast Cancer Res Treat: May 15, 2018 (Epub ahead of print).

14. Dieci MV, Frassoldati A, Generali D, Bisagni G, Piacentini F, Cavanna L, Cagossi K, Puglisi F, Michelotti A, Berardi R, et al: Tumor-infiltrating lymphocytes and molecular response after neoadjuvant therapy for HR+/HER2- breast cancer: Results from two prospective trials. Breast Cancer Res Treat 163: 295-302, 2017.

15. Denkert C, von Minckwitz G, Darb-Esfahani S, Lederer B, Heppner BI, Weber KE, Budczies J, Huober J, Klauschen F, Furlanetto J, et al: Tumour-infiltrating lymphocytes and prognosis in different subtypes of breast cancer: A pooled analysis of 3771 patients treated with neoadjuvant therapy. Lancet Oncol 19: 40-50, 2018.

16. Ohtani H, Mori-Shiraishi K, Nakajima M and Ueki H: Defining lymphocyte-predominant breast cancer by the proportion of lymphocyte-rich stroma and its significance in routine histopathological diagnosis. Pathol Int 65: 644-651, 2015.

17. Salgado R, Denkert C, Campbell C, Savas P, Nuciforo P, Aura C, de Azambuja E, Eidtmann H, Ellis CE, Baselga J, et al: Tumor-infiltrating lymphocytes and associations with pathological complete response and event-free survival in HER2-positive early-stage breast cancer treated with lapatinib and trastuzumab: A secondary analysis of the NeoALTTO trial. JAMA Oncol 1: 448-454, 2015.

18. Tsoutsou PG, Bourhis J and Coukos G: Tumor-infiltrating lymphocytes in triple-negative breast cancer: A biomarker for use beyond prognosis? J Clin Oncol 33: 1297-1298, 2015.

19. Garcia-Teijido P, Cabal ML, Fernández IP and Pérez YF: Tumor-infiltrating lymphocytes in triple negative breast cancer: The future of immune targeting. Clin Med Insights Oncol 10 (Suppl 1): 31-39, 2016

20. Pruneri G, Gray KP, Vingiani A, Viale G, Curigliano G, Criscitiello C, Láng I, Ruhstaller T, Gianni L, Goldhirsch A, et al: Tumor-infiltrating lymphocytes (TILs) are a powerful prognostic marker in patients with triple-negative breast cancer enrolled in the IBCSG phase III randomized clinical trial 22-00. Breast Cancer Res Treat 158: 323-331, 2016.

21. Ingold Heppner B, Untch M, Denkert C, Pfitzner BM, Lederer B Schmitt W, Eidtmann H, Fasching PA, Tesch H, Solbach C, et al: Tumor-infiltrating lymphocytes: A predictive and prognostic biomarker in neoadjuvant-treated HER2-positive breast cancer. Clin Cancer Res 22: 5747-5754, 2016

22. Adams S, Gray RJ, Demaria S, Goldstein L, Perez EA, Shulman LN, Martino S, Wang M, Jones VE, Saphner TJ, et al: Prognostic value of tumor-infiltrating lymphocytes in triple-negative breast cancers from two phase III randomized adjuvant breast cancer trials: ECOG 2197 and ECOG 1199. J Clin Oncol 32: 2959-2966, 2014.
23. Loi S, Michiels S, Salgado R, Sirtaine N, Jose V, Fumagalli D, Kellokumpu-Lehtinen PL, Bono P, Kataja V, Desmedt C, et al: Tumor infiltrating lymphocytes are prognostic in triple negative breast cancer and predictive for trastuzumab benefit in early breast cancer: Results from the FinHER trial. Ann Oncol 25 1544-1550, 2014

24. Hida AI, Sagara Y, Yotsumoto D, Kanemitsu S, Kawano J, Baba S, Rai Y, Oshiro Y, Aogi K, Sagara Y and Ohi Y: Prognostic and predictive impacts of tumor-infiltrating lymphocytes differ between Triple-negative and HER2-positive breast cancers treated with standard systemic therapies. Breast Cancer Res Treat 158: 1-9, 2016.

25. Loi S, Sirtaine N, Piette F, Salgado R, Viale G, Van Eenoo F, Rouas G, Francis P, Crown JP, Hitre E, et al: Prognostic and predictive value of tumor-infiltrating lymphocytes in a phase III randomized adjuvant breast cancer trial in node-positive breast cancer comparing the addition of docetaxel to doxorubicin with doxorubicin-based chemotherapy: BIG 02-98. J Clin Oncol 31: 860-867, 2013

26. Chung YR, Kim HJ, Jang MH and Park SY: Prognostic value of tumor infiltrating lymphocyte subsets in breast cancer depends on hormone receptor status. Breast Cancer Res Treat 161: 409-420, 2017.

27. Denkert C, von Minckwitz G, Darb-Esfahani S, Lederer B, Heppner BI, Weber KE, Budczies J, Huober J, Klauschen F, Furlanetto J, et al: Tumour-infiltrating lymphocytes and prognosis in different subtypes of breast cancer: A pooled analysis of 3771 patients treated with neoadjuvant therapy. Lancet Oncol 19: 40-50, 2018.

28. Jang N, Kwon HJ, Park MH, Kang SH and Bae YK: Prognostic value of tumor-infiltrating lymphocyte density assessed using a standardized method based on molecular subtypes and adjuvant chemotherapy in invasive breast cancer. Ann Surg Oncol 25: 937-946, 2018

29. Ogiya A, Yamazaki K, Horii R, Shien T, Horimoto Y, Masuda N, Inao $\mathrm{T}$, Hosoda $\mathrm{M}$, Ishida $\mathrm{N}$, Osako $\mathrm{T}$, et al: Post-relapse survival in patients with the early and late distant recurrence in estrogen receptor-positive HER2-negative breast cancer. Breast Cancer 24: 473-482, 2017

30. Yamashita H, Ogiya A, Shien T, Horimoto Y, Masuda $N$, Inao T, Osako T, Takahashi M, Endo Y, Hosoda M, et al: Clinicopathological factors predicting early and late distant recurrence in estrogen receptor-positive, HER2-negative breast cancer. Breast Cancer 23: 830-843, 2016.

31. Miyoshi Y, Shien T, Ogiya A, Ishida N, Yamazaki K, Horii R, Horimoto Y, Masuda N, Yasojima H, Inao T, et al: Differences in expression of the cancer stem cell marker aldehyde dehydrogenase 1 among estrogen receptor-positive/human epidermal growth factor receptor type 2-negative breast cancer cases with early, late, and no recurrence. Breast Cancer Res 18: 73, 2016.

32. Denkert C, Loibl S, Noske A, Roller M, Müller BM, Komor M, Budczies J, Darb-Esfahani S, Kronenwett R, Hanusch C, et al: Tumor-associated lymphocytes as an independent predictor of response to neoadjuvant chemotherapy in breast cancer. J Clin Oncol 28: 105-113, 2010.

33. Kotoula V, Chatzopoulos K, Lakis S, Alexopoulou Z, Timotheadou E, Zagouri F, Pentheroudakis G, Gogas H, Galani E, Efstratiou I, et al: Tumors with high-density tumor infiltrating lymphocytes constitute a favorable entity in breast cancer: A pooled analysis of four prospective adjuvant trials. Oncotarget 7: 5074-5087, 2016.

34. Dieci MV, Criscitiello C, Goubar A, Viale G, Conte P, Guarneri V, Ficarra G, Mathieu MC, Delaloge S, Curigliano G and Andre F: Prognostic value of tumor-infiltrating lymphocytes on residual disease after primary chemotherapy for triple-negative breast cancer: A retrospective multicenter study. Ann Oncol 25: 611-618, 2014.

35. Watanabe T, Hida AI, Inoue N, Imamura M, Fujimoto $Y$, Akazawa K, Hirota S and Miyoshi Y: Abundant tumor infiltrating lymphocytes after primary systemic chemotherapy predicts poor prognosis in estrogen receptor-positive/HER2-negative breast cancers. Breast Cancer Res Treat 168: 135-145, 2018.

36. Wang K, Shen T, Siegal GP and Wei S: The CD4/CD8 ratio of tumor-infiltrating lymphocytes at the tumor-host interface has prognostic value in triple-negative breast cancer. Hum Pathol 69: 110-117, 2017.

37. Tu MM, Rahim MMA, Sayed C, Mahmoud AB and Makrigiannis AP: Immunosurveillance and immunoediting of breast cancer via Class I MHC receptors. Cancer Immunol Res 5: 1016-1028, 2017 
38. Tsang JY, Au WL, Lo KY, Ni YB, Hlaing T, Hu J, Chan SK, Chan KF, Cheung SY and Tse GM: PD-L1 expression and tumor infiltrating PD-1+ lymphocytes associated with outcome in HER 2+ breast cancer patients. Breast Cancer Res Treat 162: 19-30, 2017.

39. Stovgaard ES, Nielsen D, Hogdall E and Balslev E: Triple negative breast cancer-prognostic role of immune-related factors: A systematic review. Acta Oncol 57: 74-82, 2018

40. Nguyen L, Tosun AB, Fine JL, Lee AV, Taylor DL and Chennubhotla SC: Spatial statistics for segmenting histological structures in H\&E stained tissue images. IEEE Trans Med Imaging 36: 1522-1532, 2017.

41. Semeraro M, Adam J, Stoll G, Louvet E, Chaba K, Poirier-Colame V, Sauvat A, Senovilla L, Vacchelli E, Bloy $\mathrm{N}$, et al: The ratio of $\mathrm{CD}^{+} / \mathrm{FOXP} 3 \mathrm{~T}$ lymphocytes infiltrating breast tissues predicts the relapse of ductal carcinoma in situ. Oncoimmunology 5: e1218106, 2016.

42. Botti G, Collina F, Scognamiglio G, Rao F, Peluso V, De Cecio R, Piezzo M, Landi G, De Laurentiis M, Cantile M and Di Bonito M: Programmed death ligand 1 (PD-L1) tumor expression is associated with a better prognosis and diabetic disease in triple negative breast cancer patients. Int J Mol Sci 18: pii: E459, 2017.

43. Kochi M, Iwamoto T, Niikura N, Bianchini G, Masuda S, Mizoo T, Nogami T, Shien T, Motoki T, Taira N, et al: Tumour-infiltrating lymphocytes (TILs)-related genomic signature predicts chemotherapy response in breast cancer. Breast Cancer Res Treat 167 39-47, 2018.

44. Kotoula V, Lakis S, Vlachos IS, Giannoulatou E, Zagouri F, Alexopoulou Z, Gogas H, Pectasides D, Aravantinos G, Efstratiou I, et al: Tumor infiltrating lymphocytes affect the outcome of patients with operable triple-negative breast cancer in combination with mutated amino acid classes. PLoS One 11: e0163138, 2016
45. Castaneda CA, Mittendorf E, Casavilca S, Wu Y, Castillo M, Arboleda P, Nunez T, Guerra H, Barrionuevo C, Dolores-Cerna $\mathrm{K}$, et al: Tumor infiltrating lymphocytes in triple negative breast cancer receiving neoadjuvant chemotherapy. World J Clin Oncol 7: 387-394, 2016.

46. Loi S: Tumor-infiltrating lymphocytes, breast cancer subtypes and therapeutic efficacy. Oncoimmunology 2: e24720, 2013.

47. Maley CC, Koelble K, Natrajan R, Aktipis A and Yuan Y: An ecological measure of immune-cancer colocalization as a prognostic factor for breast cancer. Breast Cancer Res 17: 131, 2015.

48. Qian F, Qingping Y, Linquan W, Xiaojin H, Rongshou W, Shanshan R, Wenjun L, Yong H and Enliang L: High tumor-infiltrating FoxP3 ${ }^{+} \mathrm{T}$ cells predict poor survival in estrogen receptor-positive breast cancer: A meta-analysis. Eur J Surg Oncol 43: 1258-1264, 2017.

49. Klauschen F, Müller KR, Binder A, Bockmayr M, Hägele M, Seegerer P, Wienert S, Pruneri G, de Maria S, Badve S, et al: Scoring of tumor-infiltrating lymphocytes: From visual estimation to machine learning. Semin Cancer Biol 52: 151-157, 2018.

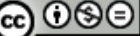

This work is licensed under a Creative Common Attribution-NonCommercial-NoDerivatives 4.0 International (CC BY-NC-ND 4.0) License. 